\title{
Infecciones invasoras por Haemophilus influenzae tipo b después de la incorporación de la vacuna conjugada al Programa Ampliado de Inmunizaciones en Chile
}

\author{
Pablo Cruces R., Alejandro Donoso F., Jorge Camacho A. y Marcela Llorente H.
}

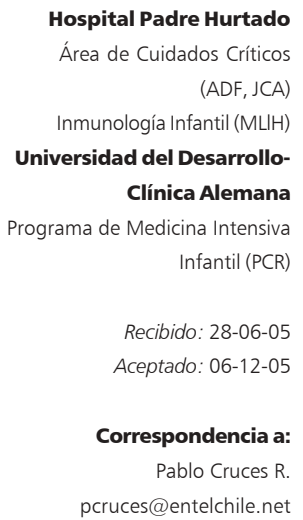

Hospital Padre Hurtado os Críticos

MLIH) Universidad del DesarrolloClínica Alemana @entelchile.net

\section{Invasive infections caused by Haemophilus influenzae type $b$ after the institution of the conjugated vaccine on the expanded programm on immunization in Chile}

After almost a decade since the introduction of Haemophilus influenzae type b (Hib) conjugate vaccines in Chile (in a 2-4-6 month schedule), Hib invasive infections have dramatically decreased, albeit they remain to occasionally produce disease in pediatric patients. We report our experience with children whom developed Hib invasive disease in children since 2000 to 2004. Medical records of children with Hib were reviewed in order to describe the epidemiology, main clinical and laboratory findings, management and complications. Twenty three patients (17 male), between 1 and 71 months (median 30 months) were identified: pneumonia (7), meningitis (4), pleuropneumonia (2), empyema (2), sepsis (2), cellulitis (2), meningitis and pleuropneumonia (1), purpura fulminans (1), miositis (1) and epiglottitis (1). No deaths were observed and four patients presented severe sequelae at hospital discharge. Twenty patients were considered vaccine failures. Hib remains as a sporadic cause of severe disease in Chile and thus for physicians should still keep it in mind. Case analysis and active surveillance are necessary to monitor the current immunization regimen.

Key words: Haemophilus influenzae type b, pneumonia, meningitis, vaccine failure, pediatrics.

Palabras claves: Haemophilus influenzae tipo b, neumonía, meningitis, falla de vacuna, pediatría.

\section{Introducción}

$\mathrm{H}$ asta hace un par de décadas las infecciones por Haemophilus influenzae tipo b (Hib) causaban aproximadamente $70 \%$ de las meningitis bacterianas agudas y eran la segunda causa de neumonía bacteriana del lactante en Chile. Así la incidencia anual de infección en Santiago reportada era de 43 casos por 100.000 habitantes bajo 5 años de edad y $80 \%$ de estas infecciones ocurrían bajo 18 meses de edad. También era un agente causal frecuente de otras patologías como artritis, epiglotitis, celulitis, pericarditis, etc ${ }^{1}$.

La introducción de la vacuna conjugada anti-Hib, que en Chile se realizó en el año 1996 con un esquema de 3 dosis (2-4-6 meses), es considerada una de las intervenciones más trascendentes en la salud pública de los últimos años ${ }^{2}$. Estudios nacionales y extranjeros confirman que el uso de esta vacuna constituye una intervención altamente efectiva, logrando una protección, con esquema de tres dosis, superior al 90\% para infecciones invasoras ${ }^{3,4}$. Sin embargo, en el concierto mundial Hib es aún responsable de la muerte de 300.000 a 400.000 niños al año, las que ocurren principalmente en países que carecen de un calendario de vacunación adecuado ${ }^{5,6}$.
Es importante destacar que en algunas poblaciones con elevadas tasas históricas de enfermedades invasoras por Hib, la portación orofaríngea de Hib continúa siendo significativa ${ }^{7-9}$. Además, recientes publicaciones nos alertan sobre el aumento de la incidencia de enfermedad invasora por Hib en los últimos años en algunos países con vacunación anti-Hib sistemática y altas coberturas ${ }^{10}$.

Consideramos de interés reportar los casos de infecciones invasoras por $\mathrm{Hib}$ registrados en nuestro centro hospitalario, destacando así la persistencia de esta bacteria, aún en niños completamente inmunizados contra Hib, la necesidad de recopilar los casos generados post introducción de la vacuna, tener un adecuado seguimiento epidemiológico y evaluar en forma continua el calendario de vacunación actual.

\section{Pacientes y Método}

Se revisaron todos los diagnósticos de alta de la Unidad de Gestión Clínica del Niño del Hospital Padre Hurtado, establecimiento público de la Región Metropolitana (Santiago) que atiende una población infantil de 0 a 4 años de 34.606 según censo del año 2003, 
ocurridos entre enero 2000 y diciembre 2004. Se pesquisaron en forma retrospectiva los pacientes en quienes se consignó al egreso el diagnóstico de enfermedad invasora por Hib en cualquiera de sus formas.

Definición: Enfermedad invasora por Hib se definió por la identificación de Hib en un sitio habitualmente estéril.

Luego se revisaron las fichas clínicas de los pacientes seleccionados para obtener los siguientes datos:

- Lugar de hospitalización: Área de Cuidados Básicos o Área de Cuidados Críticos.

- Variables biodemográficas: edad, sexo, procedencia y año de presentación.

- Antecedentes médicos: Presencia de enfermedades predisponentes a falla de vacuna, manifestaciones clínicas, sitio (s) de recuperación de Hib, disfunción de órganos y morbimortalidad asociada.

- Susceptibilidad in vitro a antimicrobianos.

- Número de dosis de vacunación anti-Hib, lo que se verificó en el carnet de su consultorio de salud y/o ficha clínica respectiva, clasificándose luego como vacunación completa o no.

Falla de vacunación fue definida como la recuperación de Hib desde sangre o LCR transcurrida una semana o más de la segunda dosis de vacuna para Hib en un paciente bajo un año de edad y después de dos semanas de la última dosis de vacuna contra Hib en pacientes de un año de edad o más ${ }^{11}$.

Identificación de Haemophilus influenzae tipo b. Las muestras clínicas fueron incubadas en agar sangre y en agar chocolate en estufa con $\mathrm{CO}_{2}$, sospechándose su presencia por el aspecto de sus colonias y la tinción de Gram. La identificación de especie se hizo mediante el kit de pruebas bioquímicas API ${ }^{\circledR N H}$ (NeisseriaHaemophilus) BioMèrieux. Todos los aislados obtenidos de sitios estériles fueron enviados al Laboratorio de Referencia del Instituto de Salud Pública, para confirmar su serotipo. Se empleó en una primera etapa antisueros de screening anticapsulares con técnica de hemaglutinación (Phadebac $\AA$, Pharmacia, Diagnostics, Uppsala Suecia) y luego la identificación del PFR del tipo $\mathrm{b}$ mediante aglutinación en lámina con antisueros DIFCO.

\section{Resultados}

Durante el periodo evaluado hubo 21.941 egresos de la Unidad de Gestión Clínica del Niño del Hospital Padre Hurtado, con el diagnóstico de enfermedad invasora por Hib en 23 de ellos $(0,1 \%)$. En el mismo período, de un total de 3.339 pacientes egresados del Área de Cuidados Críticos, 13 de ellos presentaron ese diagnóstico $(0,39 \%)$. En la Tabla 1 se muestran las principales características demográficas, co-morbilidades, localizaciones, complicaciones y resolución de los episodios.

Trece de los 23 pacientes (56\%) ingresaron directamente al Área de Cuidados Críticos. Los 10 restantes estuvieron siempre hospitalizados en el Área de Cuidados Básicos.

Epidemiología y estado vaccinal. Diecisiete pacientes $(74 \%)$ eran de sexo masculino, con una mediana de edad de 30 meses (1 - 71 meses). El 22\% de los niños tenía bajo un año de edad. Se registraron 9 casos entre los años 2000 y $2002(39 \%)$ y 14 casos entre los años 2003 y 2004 (61\%) y según el sitio de procedencia, 20 de ellos (87\%) correspondían a las comunas asignadas al hospital (Tabla 2).

Tres niños (13\%) no habían recibido esquema completo de vacuna, por tener menos de seis meses de edad.

Presencia de enfermedad de base. Cuatro pacientes $(17 \%)$ tenían antecedentes mórbidos: fístula de LCR (1) y prematurez (3), que en ningún caso fue inferior a 34 semanas de edad gestacional.

Características clínicas. La enfermedad invasora por Hib se presentó como neumonía (7), meningitis (4), pleuroneumonía (2), empiema pleural (2), sepsis (2), celulitis de pierna (2), purpura fulminans (1), meningitis + pleuroneumonía (1), miositis cervical (1) y epiglotitis (1).

En 9 pacientes (39\%) se aisló Hib simultáneamente en más de un sitio (Tabla 1).

En $21(91 \%)$ pacientes se aisló en ambos hemocultivos, en 5 pacientes LCR (22\%), en $3(13 \%)$ pacientes de líquido pleural, 2 (9\%) en tejido celular subcutáneo y uno en secreción de la región epiglótica.

La existencia de falla de órganos durante su evolución se aprecia en la Tabla 1 , destacando que 6 niños (26\%) presentaron shock séptico.

Veintiún pacientes fueron tratados desde el ingreso con cefalosporinas y dos con otros $\beta$-lactámicos, completando 7 días en el caso de celulitis y epiglotitis y 10 a 14 días en el resto de las localizaciones. En 8 pacientes $(35 \%)$, Hib fue productor de $\beta$-lactamasa.

Se realizó quimioprofilaxis de los contactos con rifampicina, según la recomendación del Departamento de Epidemiología del Ministerio de Salud, Circular No 4F/2 del 25 de enero de 1996.

Todos los pacientes fueron posteriormente referidos a evaluación por Inmunología y/o Infectología. Se realizó estudio de inmunoglobulinas séricas totales (IgG-IgM-IgA), concentración plasmática de C3 y C4, serología (ELISA) para VIH y ecografía abdominal para 


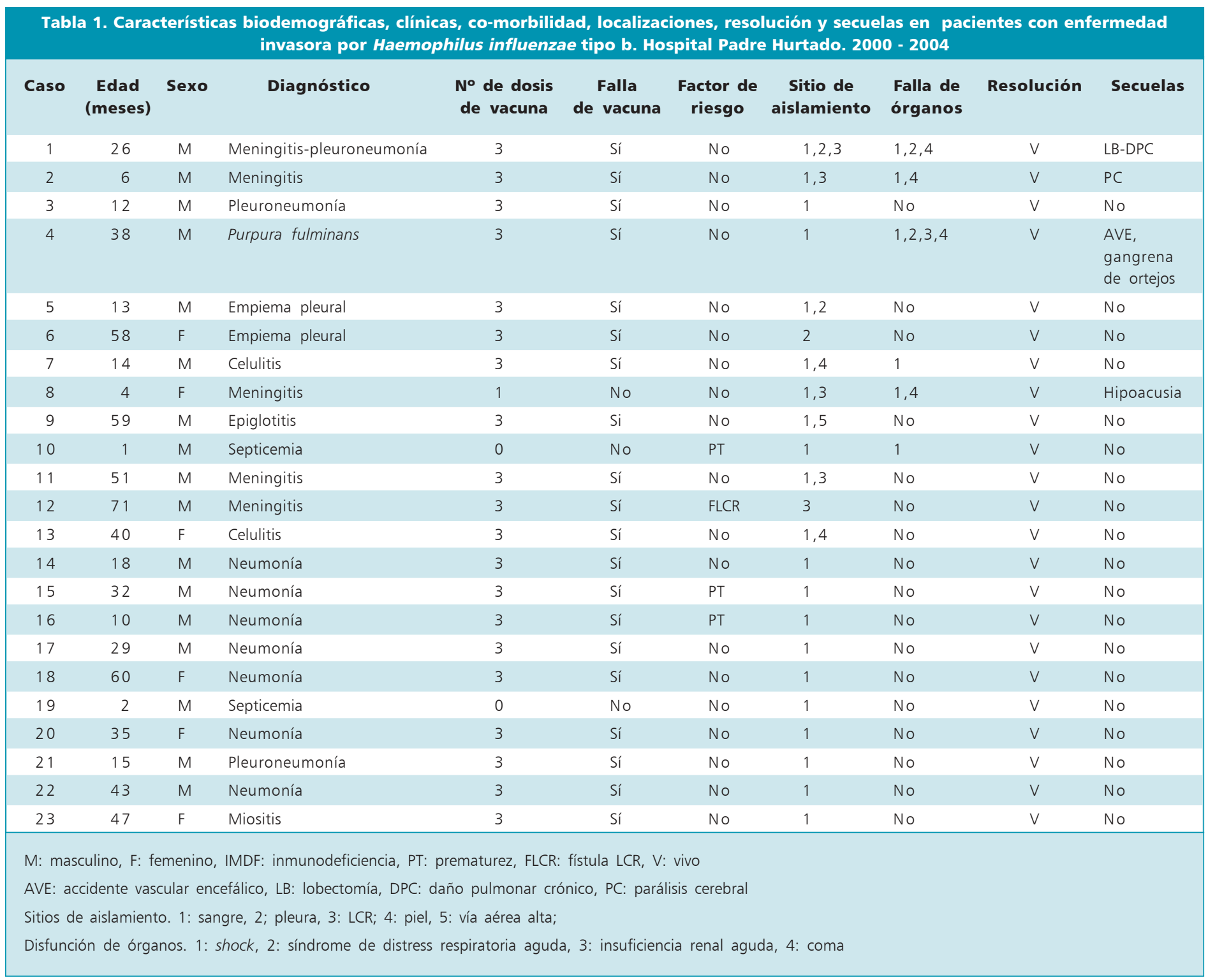

Tabla 2. Año de ocurrencia, número de casos, comuna de origen e identificación de cada paciente con diagnóstico de enfermedad invasora por Haemophilus influenzae tipo b. Hospital Padre Hurtado. 2000 - 2004

\begin{tabular}{cccc} 
Año & n de casos & $\begin{array}{c}\text { Comuna de origen } \\
(\mathbf{n})\end{array}$ & Casos $\mathbf{n}$ \\
\hline 2000 & 2 & $\mathrm{LG}(1), \mathrm{LP}(1)$ & 21,22 \\
\hline 2001 & 2 & $\mathrm{SR}(1), \mathrm{LP}(1)$ & 7,20 \\
\hline 2002 & 5 & $\mathrm{SR}(1), \mathrm{LP}(4)$ & $6,8,10,15,17$ \\
2003 & 7 & $\mathrm{SR}(1), \mathrm{O}(1), \mathrm{LG}(2), \mathrm{LP}(3)$ & $1,9,11,12,16,18,19$ \\
2004 & 7 & $\mathrm{SR}(2), \mathrm{O}(2), \mathrm{LP}(3)$ & $2,3,4,5,13,14,23$
\end{tabular}

SR: San Ramón, LG: La Granja, LP: La Pintana, OC: otra comuna descartar ausencia de bazo. Todos los resultados estuvieron dentro de rangos normales.

A todos los pacientes que habían completado su serie primaria de vacunación, se les indicó una dosis de refuerzo.

Letalidad y secuelas. No hubo pacientes fallecidos, aunque 4 niños presentaron secuelas permanentes al momento del alta: daño pulmonar crónico (1), parálisis cerebral (1), accidente vascular encefálico (1), amputación de ortejos e hipoacusia (1).

Condición de falla de vacuna. Veinte pacientes (87\%) cumplieron criterios para ser considerados como falla de vacuna. El análisis de año de vacunación y 
comuna de residencia (indirectamente refleja el lugar de aplicación de la vacuna) no permitió plantear una relación entre este proceso de vacunación y un determinado lote de vacuna o quiebre en la cadena de frío (Tabla 2).

\section{Discusión}

Un pequeño número de niños puede desarrollar una infección invasora por Hib pese a haber completado su inmunización con vacunas conjugadas anti-Hib ${ }^{12-14}$. Recientemente la incidencia de enfermedad por Hib en niños adecuadamente vacunados ha aumentado ${ }^{10,12}$, describiéndose resurgimiento de esta enfermedad en Alaska $^{8}$ y en el Reino Unido ${ }^{10,15}$. En términos generales esto puede deberse a factores propios del paciente que determinen una menor respuesta a la vacunación como también a fallas en la inmunogenicidad de la vacuna. Aunque se conocen algunos factores predisponentes como son la prematurez y la existencia de inmunodeficiencia, aunque en un porcentaje elevado de casos no se detecta ningún factor de riesgo.

El régimen originalmente recomendado de vacunación consiste en la administración de tres dosis durante el primer año de vida, más una dosis de refuerzo durante el segundo. Este esquema de 4 dosis, por razones económicas, ha sido adoptado sólo por algunos países; a pesar que la vigilancia de infecciones invasoras por Hib conducida por el Oxford Vaccine Group, the British Paediatric Surveillance Unit y el Health Protection Agency's (HPA) Communicable Diseases Surveillance Centre y el Haemophilus Reference Unit confirman la efectividad de esta intervención, la decisión es aún controversial para algunos autores $^{14}$.

Nos parece importante caracterizar la población con infecciones invasoras por $\mathrm{Hib}$ atendidas en nuestro hospital y difundir la importancia del adecuado registro y estudio de estos pacientes. Nuestra casuística incluyó principalmente niños sobre un año de edad, con calendario de vacunación completo y sin factores de riesgo asociados, cuyo foco infeccioso fue predominantemente pulmonar. De los 3 pacientes con calendario de vacunación incompleto, 2 no habían alcanzado a recibir ninguna dosis por tener menos de dos meses de edad y fueron los únicos que presentaron sepsis sin focalización de la infección. Destacamos que el perfil de nuestro grupo fue radicalmente distinto al de la era pre-vacuna, menores de 18 meses, en que se describía como foco predominantemente el meníngeo ${ }^{1}$. No habiendo en nuestra experiencia forma de predefinir factores de riesgo para contraer infecciones invasoras por Hib es necesario enfatizar la permanente importancia de tener presente este agente etiológico.
Estudios fase II de la vacuna conjugada anti-Hib concluyeron que una concentración de anticuerpos séricos anti-poliribofosfato-PRF de $0,15 \mu \mathrm{g} / \mathrm{mL}$ y 1,0 $\mu \mathrm{g} / \mathrm{mL}$ se correlacionan con protección a corto y largo plazo respectivamente, frente a infecciones invasoras por $\mathrm{Hib}^{16}$; sin embargo, éste no sería el único factor implicado ya que un estudio de seroprevalencia en niños británicos mostró que $32 \%$ de ellos tenían concentraciones inferiores a $0,15 \mu \mathrm{g} / \mathrm{mL}$ a la edad de 6 años, sin incremento de la incidencia de enfermedad en este grupo etario ${ }^{17}$. En nuestra casuística no se midió la concentración de anticuerpos por anti-PRF basales ni en la convalescencia de la enfermedad. Previamente se ha reportado en una cohorte de niños con falla de vacuna en el Reino Unido, que 30\% tendrían deficiencia de inmunoglobulinas o subclase ${ }^{11}$, aunque en un análisis ulterior, Holmes y Granoff nos muestran que no habría correlación entre falla de vacuna y las deficiencias mencionadas ${ }^{18}$. Así se ha postulado que el polimorfismo genético responsable tanto de modular la respuesta humoral, como del reconocimiento y depuración de patógenos, conferiría susceptibilidad a estas infecciones, factores probablemente independientes de la inmunogenicidad de la vacuna ${ }^{19}$.

Nuestra revisión difiere de una reciente comunicación de Villó y cols ${ }^{20}$, especialmente en cuanto a la gravedad y secuelas observadas, pues en ella, que abarcó un período de doce años, los 28 pacientes ingresados por enfermedad invasora causada por Hib (35\% con meningitis) presentaron una evolución satisfactoria y sin secuelas. Otro aspecto a destacar es que todos, a excepción de uno, se presentaron antes del inicio de la vacunación sistemática, siendo este último un paciente no vacunado ${ }^{20}$.

Esta comunicación pretende alertar en cuanto a que Hib es aún un agente que se observa en población infantil sin factores de riesgo conocidos y es causal de enfermedad grave en nuestro país, por lo que es importante mantener permanentemente un alto índice de sospecha. Su estudio y notificación son relevantes para la evaluación del esquema de vacunación actualmente utilizado. Finalmente, el hecho que las infecciones invasoras por Hib descritas en nuestra serie ocurrieron predominantemente en lactantes mayores y preescolares, a diferencia de observaciones previas a la incorporación de la vacuna al PAI en Chile, constituye un antecedente en una eventual discusión sobre el actual esquema de aplicación de la vacuna anti-Hib en el país.

\section{Resumen}

Tras nueve años desde la introducción en Chile de la vacuna conjugada contra Haemophilus influenzae 
tipo b (Hib) a las edades de 2-4 y 6 meses, las infecciones por este agente han disminuido notoriamente, pero aún son causa de morbilidad de importancia en algunos pacientes. Se reportan los niños con enfermedad invasora por Hib ocurridas entre los años 2000 y 2004. Para esto se analizaron los egresos del Hospital Padre Hurtado, consignándose epidemiología, clínica, laboratorio, terapia y complicaciones para cada paciente. Durante este período, 23 pacientes (17 varones), con una mediana de edad de 30 meses (rango 1-71 meses) presentaron enfermedad invasora por Hib. Estas se presentaron como neumonía (7), meningitis (4), pleuroneumonía (2), empiema pleural (2), sepsis (2), celulitis (2), meningitis y pleuroneumonía (1), purpura fulminans (1), miositis (1) y epiglotitis (1). No se registraron fallecimientos, pero 4 pacientes presentaron secuelas graves al momento del alta. Veinte pacientes fueron catalogados como falla de vacuna. Hamophilus influenzae b es aún un agente causal de enfermedad grave y con morbilidad asociada en nuestro país, por lo que es importante tener un alto índice de sospecha. $\mathrm{Su}$ estudio y notificación son relevantes para la evaluación del esquema de vacunación anti-Hib actualmente utilizado.

\section{Referencias}

1.- Ferreccio C, Ortiz E, Astroza L, Rivera C, Clemens J, Levine M M. A population-based retrospective assessment of the disease burden resulting from invasive Haemophilus influenzae in infants and young children in Santiago, Chile. Pediatr Infect Dis J 1990; 9: 488-94.

2.- Steinhoff M C. Haemophilus influenzae type $\mathrm{b}$ infections are preventable everywhere. Lancet 1997; 339: 1186-7.

3.- Centers for Diseases Control. Progress toward eliminating Haemophilus influenzae type $\mathrm{b}$ disease among infants and children United States, 1987-97. MMWR Morb Mortal Wkly Rep 1998; 47: 993-8.

4.- Lagos R, Horwitz I, Toro J, San Martín O, Abrego P, Bustamante C, et al. Large scale, postlicensure, selective vaccination of Chilean infants with PRP-T conjugate vaccine: practicality and effectiveness in preventing invasive Haemophilus influenzae type b infections. Pediatr Infect Dis J 1996; 15: 216-22.

5.- Watt J P, Levine O S, Santosham M. Global reduction of Hib disease: what are the next steps? Proceedings of the Meeting Scottsdale, Arizona, September 22-25, 2002. J Pediatr 2003; 143: S163-87.

6.- Sazawal S, Black R E. Pneumonia Case Management Trials Group. Effect of pneumonia case management on mortality in neonates, infants, and preschool children: a meta-analysis of community-based trials. Lancet Infect Dis 2003; 3: 547-56.
7.- Takala A K, Santosham M, Almeido-Hill J, Wolff M, Newcomer W, Reid R, et al. Vaccination with Haemophilus influenzae type $\mathrm{b}$ meningococcal protein conjugate vaccine reduces oropharyngeal carriage of Haemophilus influenzae type $\mathrm{b}$ among American Indian children. Pediatr Infect Dis J 1993; 12: 593-9.

8.- Galil K, Singleton R, Levine O S, Fitzgerald M A, Bulkow L, Getty M, et al. Re-emergence of invasive Haemophilus influenzae type $\mathrm{b}$ disease in a well-vaccinated population in remote Alaska. J Infect Dis 1999; 179: 101-6.

9.- Smith-Vaughan H C, Sriprakash K S, Leach A J, Mathews J D, Kemp D J. Low genetic diversity of Haemophilus influenzae type b compared to non-encapsulated $H$. influenzae in a population in which $H$. influenzae is highly endemic. Infect Immun 1998; 66: 3403-9.

10.- Garner D, Weston V. Effectiveness of vaccination for Haemophilus influenzae type b. Lancet 2003; 361: 395-6.

11.- Heath P T, Booy R, Griffiths H, Clutterbuck E, Azzopardi H J, Slack M P, et al. Clinical and immunological risk factors associated with Haemophilus influenzae type $\mathrm{b}$ conjugate vaccine failure in childhood. Clin Infect Dis 2000; 31: 973-80.

12.- Booy R, Heath P T, Slack M P, Begg N, Moxon E R. Vaccine failures after primary immunisation with Haemophilus influenzae type-b conjugate vaccine without booster. Lancet 1997; 349: 1197-202.
13.- Breukels M A, Spanjaard L, Sanders L A, Rijkers G T. Immunological characterization of conjugated Haemophilus influenzae type $\mathrm{b}$ vaccine failure in infants. Clin Infect Dis 2001; 32: 1700-5.

14.- Breukels M A, Jol-Van Der Zijde E, Van Tol M J, Rijkers G T. Concentration and avidity of anti-Haemophilus influenzae type b (Hib) antibodies in serum samples obtained from patients for whom Hib vaccination failed. Clin Infect Dis 2002; 34: 191-7.

15.- Heath P T, Mcvernon J. The UK Hib vaccine experience. Arch Dis Child 2002; 86: 396-9.

16.- Kayhty H, Peltola H, Karanko V, Makela $\mathrm{PH}$. The protective level of serum antibodies to the capsular polysaccharide of Haemophilus influenzae type b. J Infect Dis 1983; 147: 1100.

17.- Johnson P D, Hanlon M, Isaacs D, Gilbert G L. Differing antibody responses to Haemophilus influenzae type $\mathrm{b}$ after meningitis or epiglotitis. Epidemiol Infect 1996; 116: 21-6.

18.- Holmes S J, Granoff D M. The biology of Haemophilus influenzae type $\mathrm{b}$ vaccination failure. J Infect Dis 1992; 154: 5121-8.

19.- Kwiatkawski D. Susceptibility to infection. Br Med J 2000; 321; 1061-5.

20.- Villó N, Blanco J E, Sevilla P, Vegas E, García M, Alvarez J, et al. Enfermedad invasiva por Streptococcus pneumoniae y Haemophilus influenzae serotipo b. Estudio retrospectivo de 12 años. An Pediatr (Barc) 2004; 61: 150-5. 